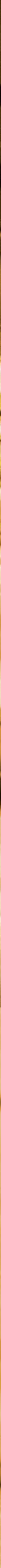




\section{Del uso de las tecnologías de la comunicación a las des- trezas en competencia mediática en las personas mayores}

The use of communication technologies to media literacy skills in elderly people

DOI: 10.15213/REDES.N12.P86

MARÍA DEL ROCÍO CRUZ DIAZ Y SARA ROMÁN GARCÍA Y FRANCISCO PAVON RABASCO

\section{ABTRACT}

Increasing life expectancy creates great challenges and difficulties in a society that must respond uniform and global population aging. Our elders have aging "full of life" and many of their capacities are still to high performance. In this article we want to approach one of the principles of active aging, training throughout life, in particular the use and development of the skills and abilities to audiovisual media and technological resources. Thus, the results of a study on media literacy in adults and older Andalusian (Spanish) by quantitative and qualitative analysis of the use of Internet and media competence level of our elders, while they are collected describes some of the advantages that may incorporate ICT in the life of the mayor: interactivity, improved self-esteem, higher levels of social participation and improving intergenerational relations. In particular some of the data obtained from the questionnaire on media competence implemented during the years 2012 and 2013, the population of people over 55 living in the Autonomous Community of Andalusia (Spain) are analyzed. Results of the research project of the Andalusian (P10SEJ-5823) "The competence of the Andalusian audiovisual citizenship. Strategies of media literacy in the digital entertainment company". Among the sample older adult participants were collected both in university classrooms of higher (university programs for older PUMs) as people without any academic links.

KEYWORDS: DIGITAL LITERACY, DIGITAL COMPETENCE, ELDERLY PEOPLE, UNIVERSITY, TRENDS AND SOCIAL WEBS 


\section{RESUMEN}

La creciente esperanza de vida plantea grandes desafíos y dificultades a una sociedad que debe dar respuesta a un envejecimiento mundial cada vez más uniforme y global. Nuestros mayores disfrutan de un envejecimiento "lleno de vida" y tienen muchas de sus capacidades al máximo rendimiento. En este artículo queremos acercarnos a uno de los principios del envejecimiento activo, la formación a lo largo de la vida, en concreto, el uso y fomento de las destrezas y habilidades con los recursos audiovisuales y los medios tecnológicos. De este modo, se recogen los resultados de un estudio sobre la competencia mediática en los adultos y mayores andaluces (españoles) mediante el análisis cuantitativo y cualitativo del uso de Internet y del nivel de competencia mediática de nuestros mayores, a la vez que se exponen algunas de las ventajas que pueden incorporar las TIC en la vida del mayor: interactividad, mejora de su autoestima, mayor nivel de participación social o mejora de las relaciones intergeneracionales. En concreto se analizan algunos de los datos obtenidos del cuestionario en competencia mediática implementado durante los años 2012 y 2013, a la población de personas mayores de 55 años residentes en la Comunidad Autónoma de Andalucía (España). Resultados obtenidos del proyecto de investigación de la Junta de Andalucía (P10SEJ-5823) "La competencia audiovisual de la ciudadanía andaluza. Estrategias de alfabetización mediática en la sociedad del ocio digital". Entre la muestra se recogen tanto adultos mayores participantes en aulas universitarias de mayores (Programas universitario para mayores PUMs) como a personas sin ningún tipo de vinculación universitaria.

PALABRAS CLAVE: ALFABETIZACIÓN DIGITAL, COMPETENCIA MEDIÁTICA, PERSONAS MAYORES, UNIVERSIDAD, TENDENCIAS Y REDES SOCIALES

\section{INTRODUCCIÓN}

De "sociedad de la información" a "sociedad del conocimiento" donde han surgido realidades como el comercio electrónico, videoconferencias, la telemedicina o las plataformas digitales. La cultura digital es eminentemente participativa, en ella los usuarios pasan de ser consumidores a ciudadanos prosumidores de contenidos, construyendo, distribuyendo y haciendo circular entre ellos dichos contenidos. Las tecnologías de la información y la 
comunicación están transformando nuestros hábitos y costumbres. La manera de trabajar, divertirnos, relacionarnos y aprender, entre otras se han visto radicalmente influenciadas por ellas. Pero ello no es suficiente y para los estudiosos, encontramos un nuevo reto al intentar lograr que "los ciudadanos sean capaces de desarrollar capacidades y estrategias que les permitan no solamente consumir medios, sino producirlos, crearlos, diseñarlos, ser agentes activos de los procesos de construcción de significados digitalizados, fomentando así prosumidores inteligentes (RAMÍREZ, RENÉS Y SÁNCHEZ, 2013: 276). Las personas mayores no han quedado ni pueden quedar al margen de este movimiento.

Las expectativas de vida en la Comunidad Europea y el resto del mundo van en aumento año tras año. Concretamente en España, debido además a la baja natalidad existente, la media alcanza los 82 años y para el 2050 se espera que sea el país más envejecido del mundo. Este mundo que envejece a marchas forzadas, se presenta cargado de retos y oportunidades. A los 60 años cada vez más personas se encuentran fuera de la considerada población activa estando aún en plenas facultades, y los que llegan a la jubilación a los 65 se encuentran todavía llenos de vida y con ganas de seguir formando parte del entramado de nuestra sociedad.

El lector de mediana edad recordará haber escuchado a personas mayores ilustradas por la sabiduría que da la experiencia, que en esta vida, hasta los 20 años se vive de ideales, de 20 a 40 años de proyectos, de 40 a 60 de realidades, de 60 a 80 de recuerdos y de 80 en adelante de milagro. El aumento del número de personas de edad avanzada está presionando en nuestro país como no lo había hecho antes. Según Pavón y Casanova (2008) aproximadamente cada mes, 36.000 personas sobrepasaban el umbral de los 65 años y ya con datos extraídos del Instituto Nacional de Estadística (INE, 2008), sobre la población mayor de 65 años que era alrededor del 17\% del total, con más de 7 millones de personas de las que aproximadamente un $25 \%$ eran octogenarias. Ya se estaba produciendo el milagro.

Años después, la publicación de la renovación padronal de 2014 (1.7.2014), indicaba que el número de personas mayores de 65 años que vivían en España alcanzaba a 8.116.553 de los que 7.727.420 son nacidos en nuestro país y 389.133 lo hicieron en el extranjero (10.700 de más de 100 años). Si consideramos los que tienen entre 60 y 65 años habría que sumar 2.268 .223 nacidos en España y 195.186 nacidos en el extranjero. Cada año España tiene más personas mayores y nos preguntamos si están integrados en la sociedad. 


\section{EDUCABILIDAD DEL MAYOR. LA AULAS UNIVERSITARIAS DE MAYORES (AUM)}

En una sociedad donde se habla permanentemente de inclusión para todos y todas, las personas que se jubilan también deben estar y sentirse integradas, nos resulta de especial interés apoyar fundamentos socioeducativos de solidez donde los ingresados en el mundo del ocio por edad y/o jubilación, puedan participar en la creación de una realidad sugerente, que dote de un nuevo sentido personal a su vida anterior y puedan entregarse apasionadamente a vivir esta etapa de su vida con la sensación de tener una misión social que cumplir y para todo eso es necesario seguir aprendiendo.

El aprendizaje a lo largo de la vida, ahora mediante el uso de nuevas tecnologías y medios de comunicación social, mantiene su indiscutida vigencia. Martínez, Cabecinhas y Loscertales (2011) han analizado que, entre otras actividades, las personas mayores acuden a Internet para sentirse más integradas socialmente, encontrando en ello beneficios o motivaciones, aunque también algunas barreras.

Desde una perspectiva formativa educacional, a partir de los años sesenta se comienza a difundir una nueva educación permanente que incide directamente en todos los formatos educativos, abriendo estructuras y niveles formativos muy dispares a quienes poseían interés por formarse. Dichos principios, promovidos una década después por la uNESCO, aspiran a generalizar la oferta de enseñanza y promover oportunidades de desarrollo a cualquier edad, en plena consonancia con la aplicación del derecho a la educación en cualquier etapa del ciclo vital. En esta línea, la Unión Europea puso en marcha entre otros el Programa "E-Europe" destinado a impulsar la sociedad de la información en la Unión Europea y años más tarde, impulsó el Programa de Aprendizaje Permanente (PAP/LLLP 2007/2013) con el objetivo de contribuir al desarrollo de la comunidad como sociedad del conocimiento avanzada y conseguir una mayor cohesión social. A resultas de estos impulsos va tomando forma una visión integral de los cambios sufridos a nivel internacional en la denominada "Agenda Digital". En este documento se concretan términos como el de alfabetización digital, que pretenden no sólo mejorar la transmisión de las competencias de aprendizaje de carácter digital sino aportar a los individuos destrezas y habilidades que les permitan integrarse plenamente en una nueva sociedad de la información.

La educación a lo largo de toda la vida es una necesidad y sólo, en la medida en que seamos cultos, seremos libres y capaces de darnos respuestas a nosotros mismos. En las últimas décadas han aparecido y promocionado, bajo el desarrollo de dicha concepción formativa, instituciones de muy diversa 
índole tales, centradas todas ellas en la promoción, formación y educación de las personas adultas y mayores, tales como: Universidades Populares, Clubs, Asociaciones de Vecinos, Centros de Educación Permanente, etc., sin dejar atrás el sistema educativo formal que potencia su permeabilidad y la Universidad, que acentúa su aperturismo con el acceso para mayores de 25 años y para mayores de 40/45 (CRUZ, MORENO-CRESPO, REBOLLEDO, 2013).

El movimiento de implantación y puesta en marcha de proyectos de formación universitaria para mayores arranca en los años 50, con una tendencia progresiva y paulatina. Los Programas Universitarios para Mayores (PUMS) hacen efectivas las funciones de la educación a lo largo de la vida como desarrollo integral de la persona, fomento de la participación y combate frente la exclusión promoviendo la evolución hacia la sociedad de la igualdad de oportunidades. 2014 fue el Año Europeo del Envejecimiento Activo y el 1 de octubre se ha conmemorado el Día Internacional de las personas mayores. En estos últimos meses del año la Asociación Estatal de Programas Universitarios de Personas Mayores (аврUм) presenta en diferentes ciudades españolas el estudio nacional. Los PuMs cuya organización y administración se encuentra, estrechamente ligada a la universidad que lo propone y lleva a término, ocupan un lugar muy importante en el desarrollo personal y social de nuestros mayores.

En España, las universidades (públicas y privadas) distribuyen las AUM entre sus distintas facultades y centros, destacando por su especial relevancia social el programa interuniversitario de Castilla y León que integra las universidades de Burgos, León, Miguel de Cervantes, Salamanca UPSA y USAL y Valladolid. La diversidad multidisciplinaria y programática en las AUMS, enriquecedora por supuesto, da paso a una necesaria reflexión en torno a criterios y toma de decisiones por parte de las universidades españolas para el impulso y gestión de un modelo de formación universitario para mayores con unos mínimos consensuados entre las universidades nacionales, que reconociesen, identificasen y consolidasen la figura del alumnado universitario mayor y su relevante papel en la sociedad actual.

\section{EL ESTUdiO DE LA COMPETENCIA MEDIÁTICA EN ADULTOS MAYORES}

A principios de siglo y tras las conclusiones del Consejo Europeo (2009) sobre un Marco estratégico para la cooperación europea en el ámbito de la educación y la formación (ET, 2020), quedaban perfiladas las competencias transver- 
sales de habilidad digital, capacidad de aprender a aprender, y la importancia de dar sentido a la capacidad de iniciativa y carácter emprendedor de todas las personas. La Nueva estrategia de la UE para el empleo y el crecimiento inteligente, sostenible e integrador, Europa 2020, publicado en 2010, otorga un papel destacado al fomento de la calidad de las iniciativas en alfabetización digital. Dos años más tarde, en 2012, la Comisión Europea redacta un nuevo documento de especial referencia en cuanto a la competencia mediática titulado Un nuevo concepto de educación: invertir en las competencias para lograr mejores resultados socioeconómicos. Para autores como Aguaded y Cruz-Díaz (2014), la competencia mediática ha sido caracterizada como una capacidad de la persona para integrar, comprender y producir mensajes, independientemente de la edad y la formación. Considerando los vertiginosos avances tecnológicos que sufre la sociedad actual, estos autores indican que la competencia mediática debe asumirse como la capacidad de aprender y desaprender el uso y producción con las tecnologías de la información y comunicación, pudiendo ser estos conocimientos transferibles y aplicables en determinadas situaciones y, la competencia mediática es fuente de conocimiento y de participación en nuestra sociedad.

Con la apuesta internacional y, en especial, europea de facilitar y promocionar estrategias tanto a nivel educativo como social que permitan el aprendizaje activo y permanente en los entornos digitales, comienzan a surgir importantes referentes de estudios e investigaciones (FERRÉs, 2006, 2007), que nos acercan a un nuevo concepto de comunicación audiovisual en todas sus dimensiones, nos referimos a la "competencia mediática". Se define el concepto de "competencia clave" o "competencia básica", como un conjunto multifuncional y transferible de conocimientos, destrezas y actitudes. En relación al desarrollo eficaz de las destrezas comunicativas y la adquisición de competencias en comunicación audiovisual supone "la capacidad de un individuo para interpretar y analizar desde la reflexión crítica las imágenes y los mensajes audiovisuales y para expresarse con una mínima corrección en ámbito comunicativo" (2007: 102). De esta forma, la "competencia en comunicación" se ve inmersa en un entorno digital y de consumo mediático en ambientes de ocio y tiempo libre gran utilidad para alcanzar objetivos audiovisuales y, que han de ser aprendidas y aplicadas con herramientas digitales. En este sentido, se hace patente la necesidad de formación en competencia mediática "que no se adquieren por el consumo diario de los medios, como muchos hasta ahora han pensado, sino que es imprescindible una formación crítica en alfabetización audiovisual y mediática, entendiendo estas como las destrezas, habilidades, actitudes y aptitudes mínimas, para poder interpre- 
tar el aluvión de imágenes y contenidos mediáticos y telemáticos en habitual social y personal" (AGUADED, 2009: 7).

En nuestro país, con objeto de profundizar en el estudio de la competencia mediática, un grupo de expertos liderados por Ferrés, Matilla y Aguaded (2011) y por investigadores e investigadoras de reconocido prestigio de otras nacionalidades, emprendieron una experiencia pionera. El proyecto titulado Competencia Mediática, Investigación sobre el grado de competencia de la ciudadanía en España, contó con el apoyo del Ministerio de Educación en España logrando medir el nivel de competencia mediática de la ciudadanía en España (AGUADED y CRUZ-DíAZ, 2014). Interesados por la formación ante el futuro más cercano de los ciudadanos adultos y mayores, en enero de 2015 y con duración prevista hasta mediados de 2016, se ha iniciado un Proyecto europeo multilateral que trata de fomentar el envejecimiento activo entre la población de mayores de 55 años mediante una metodología basada en la educación de igual a igual, formación entre pares, consistente en que personas cualificadas y con motivación (educadores entre iguales) organizan actividades educativas con sus iguales (personas de la misma edad, clase social o similares capacidades) con el objeto de desarrollar habilidades y ampliar conocimiento. La utilización de esta metodología parece resultar eficaz ya que se observa en los mayores una actitud y comportamiento más favorable, al identificarse con el educador. Se pretende llegar al menos a unas 20.000 personas mayores. La formación se realizará en pequeños grupos presenciales o mediante plataformas on-line.

Nos centraremos en el estudio de la Competencia Mediática y sus dimensiones en las Personas Mayores que, siguiendo a Ferrés y Piscitelli (2012), hace referencia al dominio de conocimientos, destrezas y actitudes relacionados con seis dimensiones básicas que tienen que ver con el ámbito de participación como personas que reciben mensajes e interaccionan con ellos (ámbito del análisis) y como personas que producen mensajes (ámbito de la expresión). Estas seis grandes dimensiones son: el lenguaje, la tecnología, la ideología y los valores, la producción y programación, la recepción e interacción y la dimensión estética.

\subsection{METODOLOGÍA E INSTRUMENTO}

La investigación realizada presenta una naturaleza cualitativa e intenta responder a dos grandes cuestiones: ¿está presente la competencia mediática entre las destrezas y habilidades que desarrollan los adultos y mayores en 
su día a día?, ¿cómo contribuye el paso por programas universitarios para personas adultas en desarrollo de la competencia mediática? y ¿qué relación puede establecerse con las seis dimen $\neg$ siones establecidas por Ferrés y Piscitelli (2012) para la mencionada competencia en los mayores andaluces?

Asimismo, a través de esta investigación damos respuesta a una de las tareas del Proyecto I+D La enseñanza obligatoria ante la competencia en comunicación audio-visual en un entorno digital (EDU2010-21395-C03-03) acerca de la evaluación de la competencia mediática con adultos y mayores.

El procedimiento seguido ha sido el siguiente:

1. Localización de las disposiciones europeas y normativa/recomendaciones que regula la formación permanente y la educación de los mayores en la comunidad autónoma en cuanto que fomentamos un envejecimiento activo.

2. Tratamiento de la información hallada a través del programa ATLAS.ti.

3. Análisis de los objetivos, contenidos y criterios del uso y la formación de los mayores en todas las provincias de la comunidad autónoma andaluza, hayan o no pasado por los Programas universitarios para personas mayores.

4. Establecimiento de relaciones entre los elementos curriculares y las dimen $\neg$ siones de la competencia mediática establecidas por Ferrés y Piscitelli (2012): Lenguajes, Tecnología, Procesos de interacción, Procesos de producción y difusión, Ideología y valores, y Estética, teniendo en consideración los dos ámbitos que componen cada una de ellas -el análisis de contenido y la expresión-.

La muestra de población de este estudio es de 479 personas mayores de 55 años pertenecientes a la comunidad autónoma andaluza, con una media de 60 personas por provincia. En Andalucía hay más mujeres que hombres de 55 años; la muestra de este estudio respeta la proporcionalidad poblacional por sexo. Del total de la población muestreada, 245 formaban parte de alguno de los Programas Universitarios de Mayores de las diferentes universidades andaluzas.

Descripción de la herramienta: El cuestionario implementado incluye 28 preguntas. Las 11 primeras cuestiones son de carácter socio-demográfico (edad, sexo, nivel de estudios, etc.); las restantes se refieren a diferentes dimensiones de la competencia mediática, tal como podemos ver en Contreras, Marfil y Ortega (2014: 133). 
De estos últimos ítems hemos seleccionado 6 para este estudio, uno por cada dimensión, al resultar significativos los resultados en su análisis y cruce con otras variables y por tener equivalentes ítems en el cuestionario de adultos, con el fin de estudiar relaciones en estudios posteriores.

\subsection{ANÁLISIS Y RESULTADOS}

El análisis que se presenta a continuación está organizado en torno a las 6 dimensiones anteriormente citadas de la Competencia Mediática en mayores, y los resultados corresponden a los ámbitos de contenido específico de los ítems elegidos para análisis y contraste, por su significatividad en los resultados obtenidos.

TABLA 1 ITEMS DEL CUESTIONARIO ELEGIDOS PARA ANÁLISIS

\begin{tabular}{|l|l|}
\hline $\begin{array}{l}\text { DIMENSIONES } \\
\text { de la Competencia } \\
\text { Mediática }\end{array}$ & Preguntas del cuestionario elegidas para análisis \\
\hline LENGUAJE & P. 13. En caso de que utilice internet, ¿para qué lo hace? \\
\hline TECNOLOGIA & P. 15. ¿Con qué aplicaciones informáticas ha trabajado? \\
\hline $\begin{array}{l}\text { PRODUCCION Y } \\
\text { PROGRAMACION }\end{array}$ & $\begin{array}{l}\text { P. 19. ¿Antes de introducir sus datos personales (tarjeta de } \\
\text { crédito, correo electrónico, teléfonos, etc.) en internet, a la } \\
\text { hora de hacer una compra, descargarse algún programa, o } \\
\text { registrarse en un servicio electrónico...? }\end{array}$ \\
\hline $\begin{array}{l}\text { RECEPCION E } \\
\text { INTERACCION }\end{array}$ & $\begin{array}{l}\text { P. 20. ¿Ha utilizado internet para comunicarse con las } \\
\text { autoridades públicas? }\end{array}$ \\
\hline $\begin{array}{l}\text { IDEOLOGIA Y } \\
\text { VALORES }\end{array}$ & $\begin{array}{l}\text { P. 24. ¿Utiliza usted algún medio (e-mail, blog, etc.) para } \\
\text { mandar mensajes, o realizar acciones, que contribuyan a la } \\
\text { mejora del entorno social en el que vive? }\end{array}$ \\
\hline ESTETICA & $\begin{array}{l}\text { P. 25. A la hora de decidirse por un medio ¿tiene en cuenta } \\
\text { sólo lo que comunica o en esa elección le da valor a los } \\
\text { aspectos formales y estéticos? }\end{array}$ \\
\hline
\end{tabular}

FUENTE: ELABORACIÓN PROPIA

3.2.1. el uso de las tecnologías por parte de los mayores. el LENGUAJE MULTIMEDIA

Para el análisis de la dimensión lenguaje, que trata de estudiar la habi- 
lidad de los mayores para interactuar de manera significativa con medios, con el objetivo de expandir sus capacidades mentales y también la capacidad de manejo de las innovaciones tecnológicas para una mejor comunicación multimedial, hemos escogido el ítem $\mathrm{n}^{\circ} 13$ (Ver gráfico 1). La situación actual según respuestas se refleja en el siguiente gráfico:

GRÁFICO 1. P. 13. RELACIÓN EDAD-UTILIZACIÓN INTERNET

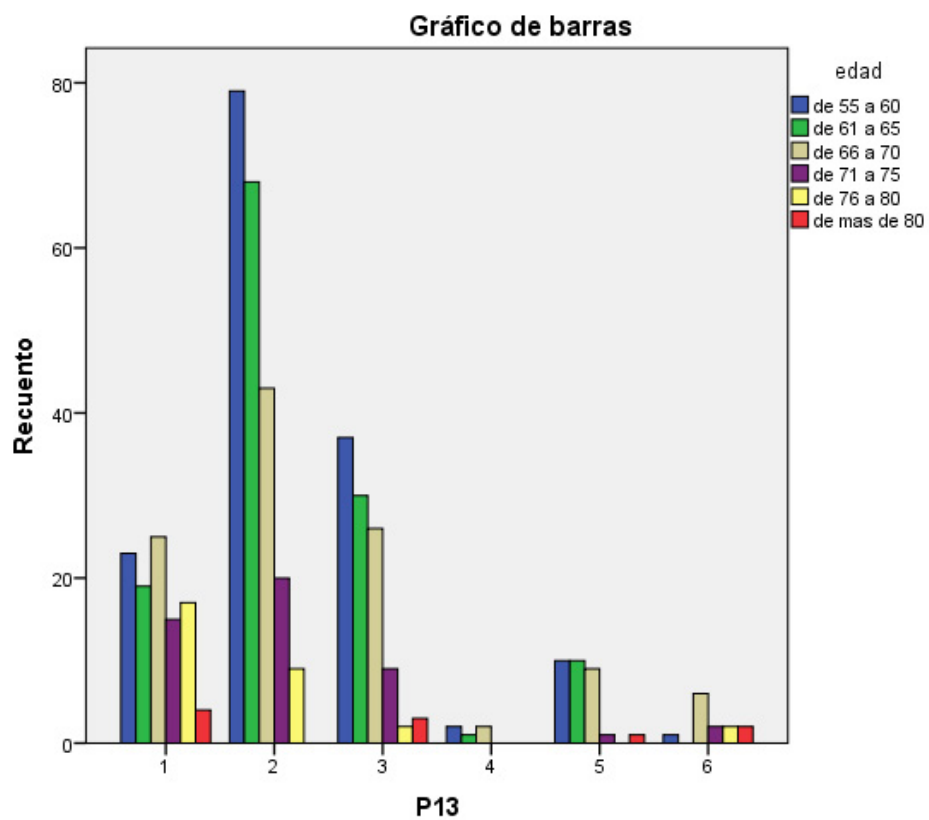

FUENTE: ELABORACIÓN PROPIA

Puede observarse cómo las actividades en la web (2), comunicación con otros (3) y consultas de bancos (5), descienden a medida que aumenta la edad; siendo la actividad más notable la consulta de información en la web (2) y la comunicación con otras personas (3). De entre los valores estadísticos destacar la relación entre edad y uso de dicho medio resulta ser significativa mediante los contrastes Chi-cuadrado y razón de verosimilitud con un coeficiente de contingencia de 0.35 .

Los mayores, como todos los ciudadanos, navegan por todas las webs de Internet, pero cada vez hay más portales específicos para mayores desde los 
cuales dirigirse a bases muy amplias de información que interesan especialmente a este colectivo. Se han detectado como posibles problemas para el uso de Internet en mayores el coste económico de los equipos o las dificultades para dedicar tiempo y dinero a cursos de formación. En consonancia con esto, al cruzar los datos sobre percepción económica y distintos usos de Internet, los resultados indican:

\section{GRÁFICO 2. P.13. RELACIÓN INGRESOS-UTILIZACIÓN INTERNET}

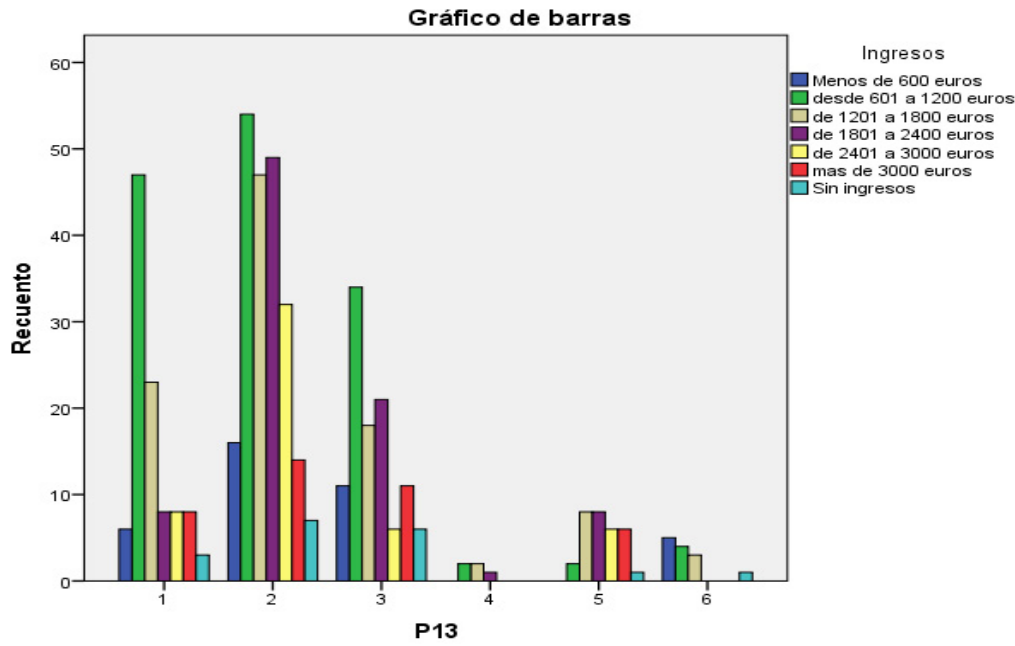

FUENTE: ELABORACIÓN PROPIA

El diagrama nos indica que el modo en que usan Internet los mayores de 55 años está en relación con los ingresos mensuales que perciben; utilizan más internet para la búsqueda o consulta de información en webs (2) los que perciben entre 600 y $2400 €$ mensuales. También realizan más contactos para comunicarse con amigos, colegas $u$ otras personas (3) los que reciben entre 600 y $1200 €$ al mes. Y además, la relación entre ingresos mensuales y utilización de Internet resulta ser significativa mediante el contraste de Chicuadrado con un coeficiente de contingencia de $35.8 \%$.

El diagrama (Ver Gráfíco 3) indica que los mayores de 55 años con estudios secundarios o universitarios utilizan más internet para la búsqueda o consulta de información en webs (2) y realizan más contactos para comunicarse con amigos, colegas u otras personas (3) que los que no tienen estudios 
o sólo estudios primarios. Igualmente, realizan más consultas en bancos (5) aquellos que tienen estudios universitarios. Y además, la relación entre nivel de estudios y uso de dicho medio resulta ser altamente significativa mediante el contraste Chi-cuadrado con un coeficiente de contingencia de $47.1 \%$.

GRÁFICO 3 P 13. RELACIÓN ESTUDIOS-UTILIZACIÓN INTERNET

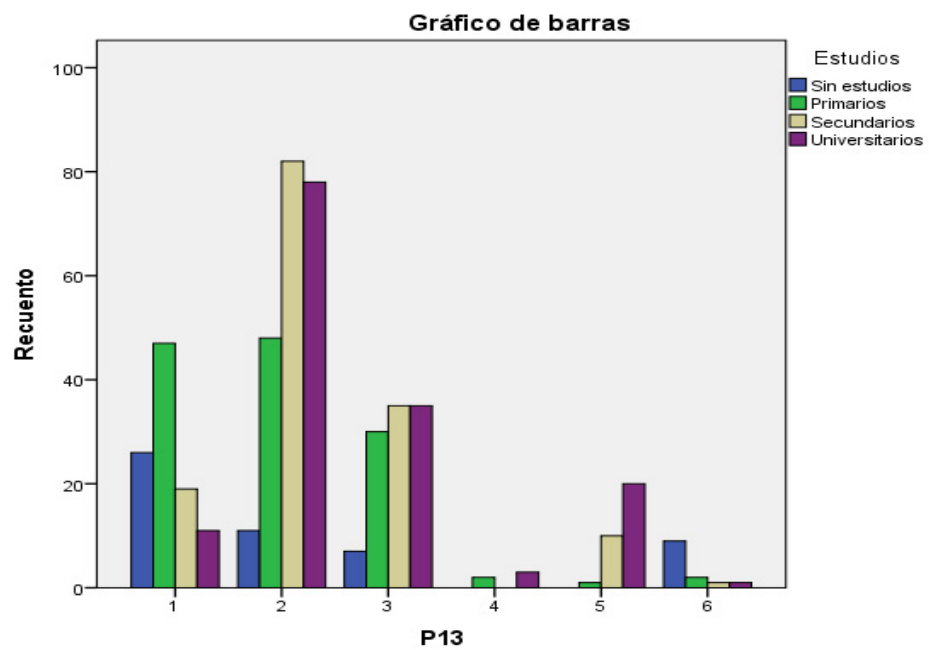

FUENTE: ELABORACIÓN PROPIA

En relación a los contenidos abordados actualmente, los aspectos relacionados con vida saludable o acomodación psicológica a la situación de inactividad laboral, etc., son considerados como ineludibles en estas aulas, tanto por parte de los responsables, como del alumnado, que demanda explícitamente su tratamiento. Por otro lado, hay cierto número de asignaturas que se centran en el estudio del entorno cultural, arquitectónico, literario, empresarial, etc., de la zona geográfica de influencia de la universidad en cuestión.

¿Pero qué ocurre con otros contenidos o herramientas que tanta utilidad podrían tener para el desenvolvimiento y adaptación al mundo presente en los mayores, como es el conocimiento de Internet? Macías y Manresa (2013) han estudiado la e-inclusión para conocer cuáles son las motivaciones que hacen que los mayores se matriculen en programas universitarios y se interesen con los temas relacionados con las TIC.

Gutiérrez y Tyner (2012) abordan el papel que le corresponde a la educa- 
ción y nos advierten del peligro de reducir la educación mediática al desarrollo de la competencia digital y ésta a su dimensión más tecnológica e instrumental, centrándose en el manejo de dispositivos y programas, olvidando las actitudes y los valores.

GRÁFICO 4 P. 13. RELACIÓN PERTENENCIA O NO A AUM-UTILIZACIÓN DE INTERNET

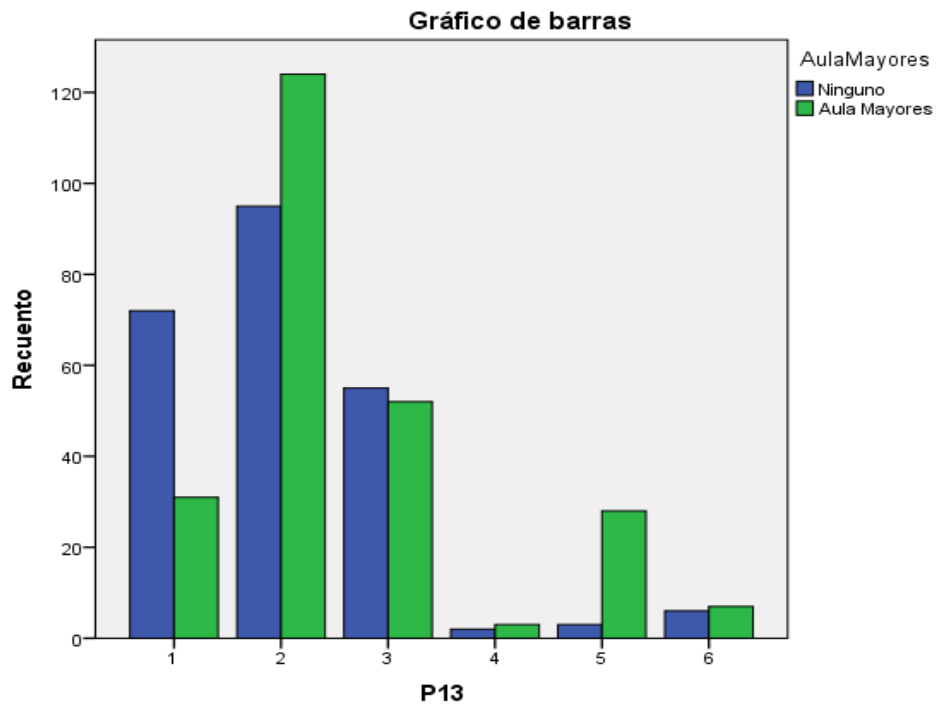

FUENTE: ELABORACIÓN PROPIA

Los mayores de 55 años que están en el aula de mayores utilizan más internet para la búsqueda o consulta de información en webs (2) que los que no pertenecen a ella. También realizan más consultas de bancos (5). Las pruebas de significación Chi-cuadrado arrojan un coeficiente de contingencia $27.9 \%$ indicando que el pertenecer al aula de mayores sí está relacionado con el uso que se le da a internet.

3.2.2. INDICADORES DE LA DIMENSIÓN TECNOLÓGICA DE LA COMPETENCIA MEdí́tica

Con relación al uso de herramientas específicas de Internet por parte 
de las personas mayores, e indagando en las aplicaciones informáticas que conocen y/o utilizan nuestros mayores (ver Tabla 1, P. 15), tendríamos que añadir que, tradicionalmente, los mayores en España no parecían muy interesados por las novedades que ofrecía la tecnología, pero hoy la situación va cambiando y nos preguntamos: $¿$ Es la jubilación un buen momento para aprender informática? Villaplana (2013), realizó un importante trabajo con el objetivo de conocer si las personas mayores consideran que la etapa de la jubilación es un momento óptimo para aprender dicha materia. Utilizando datos de la Encuesta de Condiciones de Vida de las Personas Mayores (2010) realizada por el IMSERSO a personas de más de 65 años, nos presentó tres categorías de usuarios: 1) personas que sabían informática antes de la jubilación, usuarios-pre $(6,79 \%)$, 2) los que la aprendieron después de la jubilación, usuarios-post (2,76 \%) у 3 ) los que nunca han mostrado interés, no usuarios (90,45\%). En comparación con los no usuarios, los usuarios pre o post suelen ser en mayor medida hombres, de 65-69 años, con estudios secundarios o superiores, con ingresos superiores a $900 € /$ mes y combinan la informática con otras actividades como turismo, deporte y voluntariado.

Sin embargo, ese perfil no responde en cuestión de género a los resultados obtenidos en nuestro trabajo, referido a la población de Andalucía:

GRÁFICO 5. P.15. RELACIÓN SEXO-APLICACIONES INFORMÁTICAS UTILIZADAS.

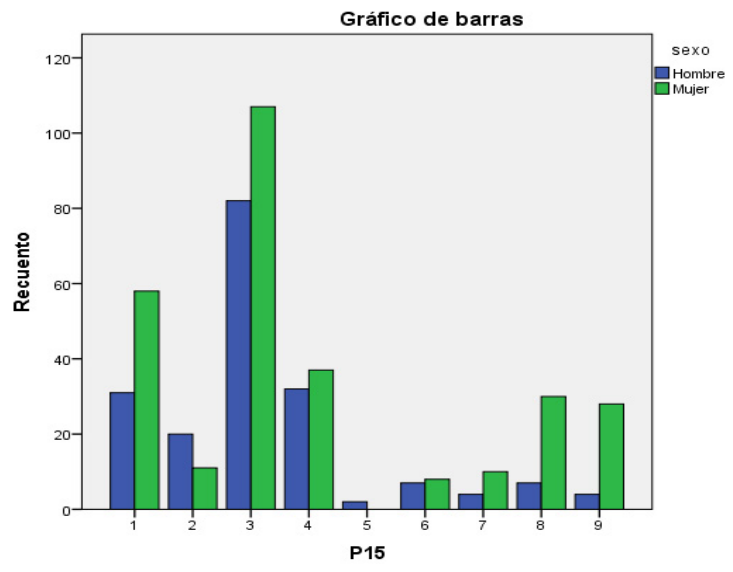

FUENTE: ELABORACIÓN PROPIA

El gráfico muestra que en mayores de 55 años, aunque hay más mujeres 
que hombres que no utilizan las aplicaciones informáticas (1), de los usuarios, utilizan más las herramientas de internet las mujeres, sobre todo el correo electrónico (3), el programa Word (4), Photoshop o Picasa (6), Messenger (7) y sobre todo Facebook (8).Y además, la relación entre sexo y uso de aplicaciones informáticas resulta ser significativa mediante el contraste Chicuadrado con un coeficiente de contingencia de $25.0 \%$.

Desde el año 1994, en que se iniciaron las actividades universitarias dirigidas a mayores en Granada, hasta el curso 1999-2000 que se iniciaron en la Universidad de Huelva, se han ido instalando estos servicios en las distintas Universidades andaluzas. Nos hacemos ahora las preguntas que muchos de vosotros o quizás de nuestros mayores se hacen cuando se enfrentan con la oferta de un programa universitario para mayores (PUMs): ¿qué puede aportar la educación a las personas mayores? En principio y generalizando, lo mismo que a cualquier otra persona no mayor: desarrollo personal, posibilidades de encuentro consigo mismo y con el otro, participación social activa... García Arroyo (2001) apunta algunas de las posibles acciones, que al participar en programas educativos, pueden generar mayor "calidad de vida» a sus participantes, en este caso, personas mayores: desarrollo de actividad corporal; ejercitar y estimular a través de situaciones diversas, la actividad mental y social como requisito imprescindible para una vejez satisfactoria; desarrollar hábitos en torno a una alimentación y nutrición adecuada; entrenarse en un estilo de vida relajado aprendiendo a minimizar las situaciones difíciles o de riesgo... (Ver Gráfica 6)

GRÁFICO 6. P. 15. RELACIÓN NIVEL DE ESTUDIOS- APLICACIONES INFORMÁTICAS UTILIZADAS.

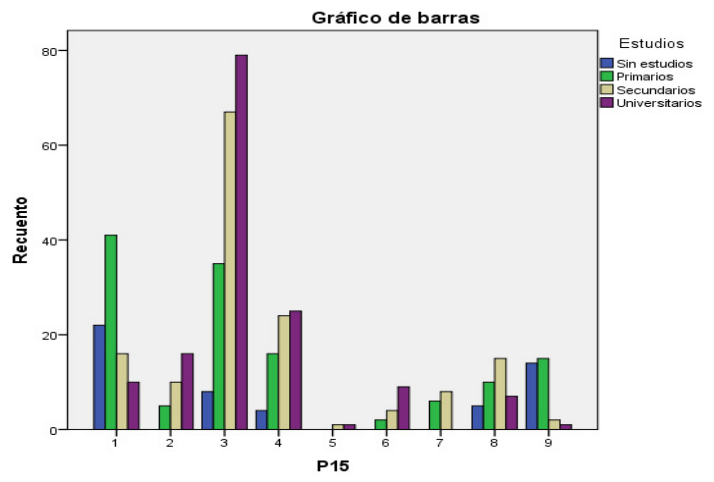

FUENTE: ELABORACIÓN PROPIA 
El diagrama de barras nos indica que los mayores de 55 años, dependiendo del nivel de estudios, utilizan más las herramientas de internet si tienen estudios de más nivel sobre todo el correo electrónico (3), el programa Word (4), Photoshop o Picasa (6), y menos Facebook (8) Y además, en el contraste de Chi-cuadrado, resulta ser significativo el nivel de estudios cuando lo relacionamos con el mayor o menor uso del correo electrónico, el programa Word o, Photoshop, Picasa o Facebook, con un coeficiente de contingencia de $48.6 \%$.

El simple hecho de acudir a recibir clases en un centro universitario ya es considerado por muchos mayores como una forma de participación activa en la sociedad, como puede ser la de sus nietos o hijos que estén estudiando una carrera. Pero, además, el efecto sobre muchos de ellos es el de sentirse personas valiosas que tienen todavía algo que aportar, y es por ello, que toman iniciativas en la línea del asociacionismo, ya indicado, o en la de la participación personal o grupal en las distintas iniciativas de sus ciudades respectivas.

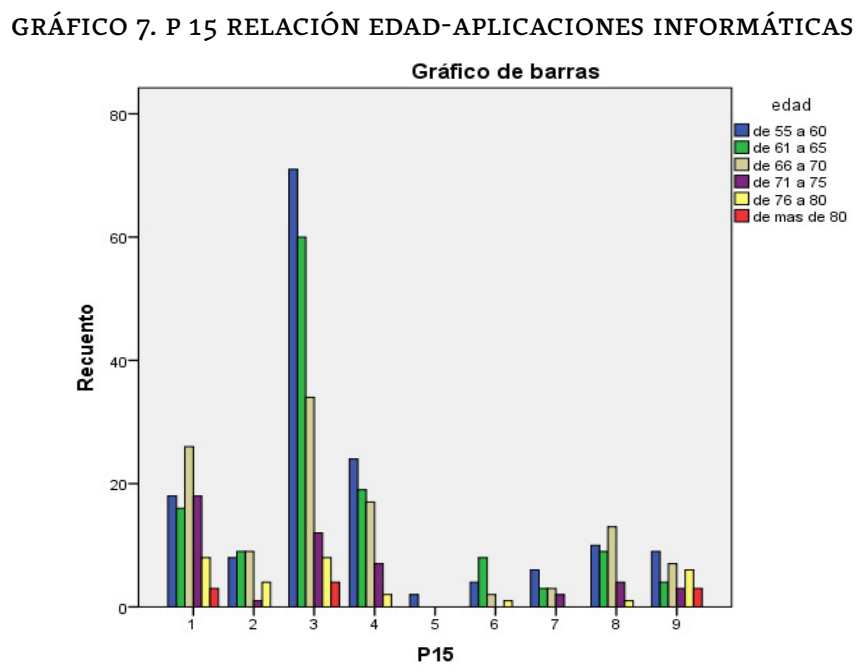

FUENTE: ELABORACIÓN PROPIA

El diagrama de barras nos indica que los mayores de 55 años utilizan más internet a través del correo electrónico (39.46\%) (3) y el programa Word y OpenOffice (14,41\%) (4), descendiendo su uso a medida que aumenta la edad. Curioso resulta ver que Facebook (8) es más usado por el grupo de edad de 66-70 años que por el 55-60. El contraste Chi-cuadrado, con un coeficiente de contingencia $36.3 \%$, indica que es muy significativa la edad con relación 
al mayor o menor uso del correo electrónico, el programa Word o Facebook.

Con el extraordinario aumento de la población mayor, nos preguntamos: ¿cómo se sostendrán las demandas de servicios adicionales que requieren las personas mayores? ¿Habrá suficientes recursos para atención a los mayores, tales como pensiones, servicios sanitarios y sociales? Quizá la teleasistencia se convierta en una solución para algunos de estos problemas; nuevos equipos que permitan dotar al mayor de mejores herramientas para tener la posibilidad de permanecer durante más tiempo viviendo solo, pero con garantías de control y calidad de vida (pAvón, 2000). Más tiempo de vida independiente donde la persona ejerza el poder de decisión sobre su propia existencia y participe activamente en la vida de su comunidad, conforme al derecho al libre desarrollo de la personalidad. Nos estamos refiriendo a todo ese conjunto de posibilidades que se les presenta a una persona que conoce cómo acceder a la información y a los servicios que se les ofrecen desde una pantalla de TV o desde el monitor de un ordenador (PAVón y CASANOVA, 2006).

\subsubsection{INDICADORES DE LA DIMENSIÓN PROdUCCIÓN Y PROGRAMACIÓN DE LA COMPETENCIA MEDIÁTICA}

Algunos indicadores de esta dimensión implican capacidad de seleccionar mensajes significativos, apropiarse de ellos y transformarlos para producir nuevos significados así como capacidad de compartir y diseminar información, a través de los medios tradicionales y de las redes sociales, incrementando la visibilidad de los mensajes, en interacción con comunidades cada vez más amplias.

Frecuentemente en España, en el pasado, se ha considerado a las personas mayores como un colectivo desvinculado ya de los circuitos de producción y consumo, considerando en cambio al ciudadano de mediana edad, y fundamentalmente al juvenil, como el más preparado para la aceptación de nuevos adelantos y el más propenso al gasto; sin embargo, los datos que tenemos de Europa nos hablan de que los mayores poseen la mitad del poder adquisitivo de la población total y se muestran receptivos a las ofertas publicitarias. 
GRÁFICO 8. P. 19 RELACIÓN CONFIANZA EN WEBS -EDAD

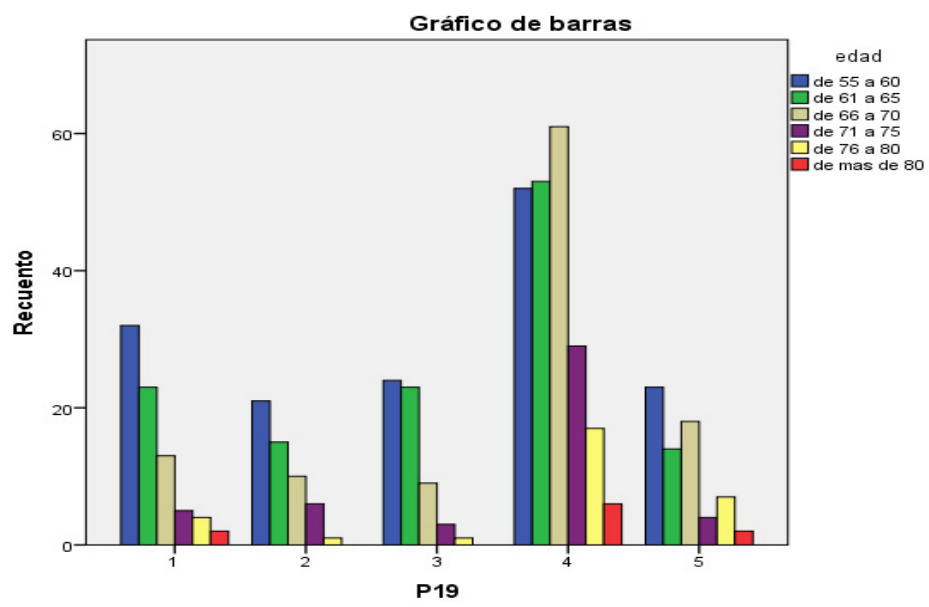

FUENTE: ELABORACIÓN PROPIA

Los mayores de 55 años, antes de introducir sus datos personales (tarjeta de crédito, correo electrónico, teléfonos, etc.) en internet, a la hora de hacer una compra, descargarse algún programa, o registrarse en un servicio electrónico, la mayoría no confiaría en ningún sitio (4) y crece esa desconfianza con la edad; mientras que las opciones de respuesta: Me dejo llevar por el aspecto profesional del sitio (1), Confío en mi instinto personal (2), descienden a medida que se cumplen años. El contraste Chi-cuadrado, con un coeficiente de contingencia $\mathbf{2 6 . 2} \%$ indica que es significativa la edad en relación a este tema.

Hoy, cuatro de cada diez internautas mayores de 60 años ha comprado alguna vez online. Según el estudio de opinión «El abuelo internauta» que ha elaborado el portal de ofertas Club Oferting (www.cluboferting.com) el $41 \%$ de los internautas españoles que tienen más de 60 años se consideran usuarios activos de internet, ya que no sólo usan la red para buscar información, sino que han hecho alguna compra online. La contratación de viajes, la compra de entradas para espectáculos y la adquisición de artículos de electrónica centran la mayor parte de las acciones de compra online de este tipo de usuarios. 
GRÁFICO 9. P.19. RELACIÓN CONFIANZA EN WEBS-INGRESOS

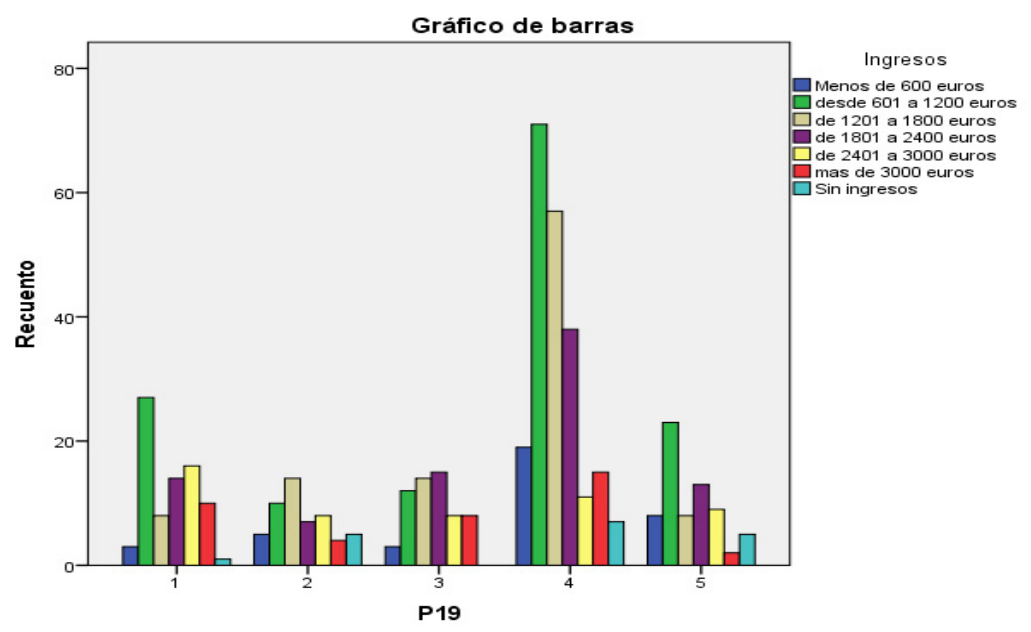

FUENTE: ELABORACIÓN PROPIA

Antes de introducir sus datos personales (tarjeta de crédito, correo electrónico, teléfonos, etc.) en internet, a la hora de hacer una compra, descargarse algún programa, o registrarse en un servicio electrónico dependiendo de su nivel de ingresos, la mayoría de los encuestados responde que no confiaría en ningún sitio (4) y esa desconfianza es mayor en los que perciben entre 600 y $1200 €$, descendiendo en cambio cuando aumenta el nivel de ingresos. El contraste de Chi-cuadrado, con un coeficiente de contingencia de $32.0 \%$, indica que es significativo el nivel de ingresos en relación a la confianza en la utilización de la web para ese tipo de operaciones.

Ya Castells (2001) anunciaba que «en la primera década del siglo XXI se espera un crecimiento más rápido del comercio electrónico en Europa que en Estados Unidos» (2001, 81-82). También en esto los mayores están llevando un ritmo bastante acelerado, y el grupo de internautas de gente mayor es el que más rápidamente crece en todo el mundo, pues tienen tiempo, dinero y múltiples intereses (VILAPLANA, 2013).

\subsubsection{INDICADORES DE LA DIMENSIÓN RECEPCIÓN E INTERACCIÓN DE LA COMPETENCIA MEDIÁTICA}

Esta dimensión de la competencia mediática se refiere a la actitud activa 
en la interacción con las pantallas, entendidas como oportunidad para construir una ciudadanía más plena, un desarrollo integral, para transformarse y para transformar el entorno así como conocimiento de las posibilidades legales de reclamación ante el incumplimiento de las normas vigentes en materia audiovisual, y actitud responsable ante estas situaciones.

GRÁFICO 10 P. 20. RELACIÓN COMUNICAR AUTORIDADES-ESTUDIOS

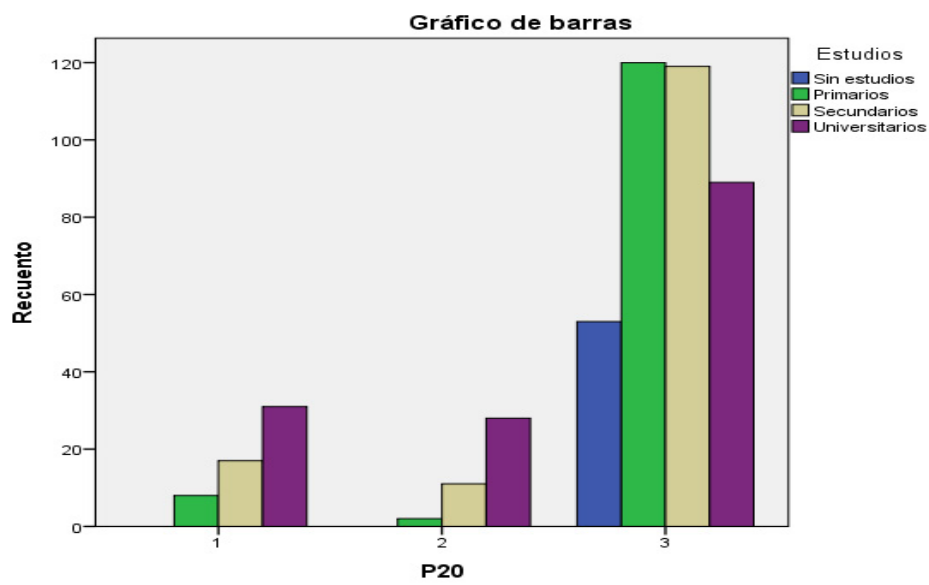

FUENTE: ELABORACIÓN PROPIA

La mayoría de los mayores de 55 años ante la pregunta: ¿Ha utilizado Internet para comunicarse con las autoridades públicas? responde, no nunca (3). Pero la mayoría de las personas que lo han utilizado en el último año (1) o en los últimos 3 meses (2), tiene estudios universitarios. El coeficiente de contingencia de $34.3 \%$ en el contraste de Chi-cuadrado nos indica que es significativa la relación entre la utilización de Internet para reclamar/comunicar con las autoridades y el nivel de estudios. Permanecer "conectado", seguir sintiéndose joven y alerta, agruparse, compensar un hándicap, todo esto responde a un temor generalizado: la muerte social, el «apartheid generacional».

\subsubsection{INDICADORES DE LA DIMENSIÓN IDEOLOGÍA Y VALORES DE LA COMPETENCIA MEDIÁTICA}

Esta dimensión está relacionada con la capacidad de aprovecharlas nuevas herramientas comunicativas para transmitir valores y para contribuir a la 
mejora del entorno, desde una actitud de compromiso social y cultural.

GRÁFICO 11 RELACIÓN DIFUSIÓN VALORES-NIVEL ESTUDIOS

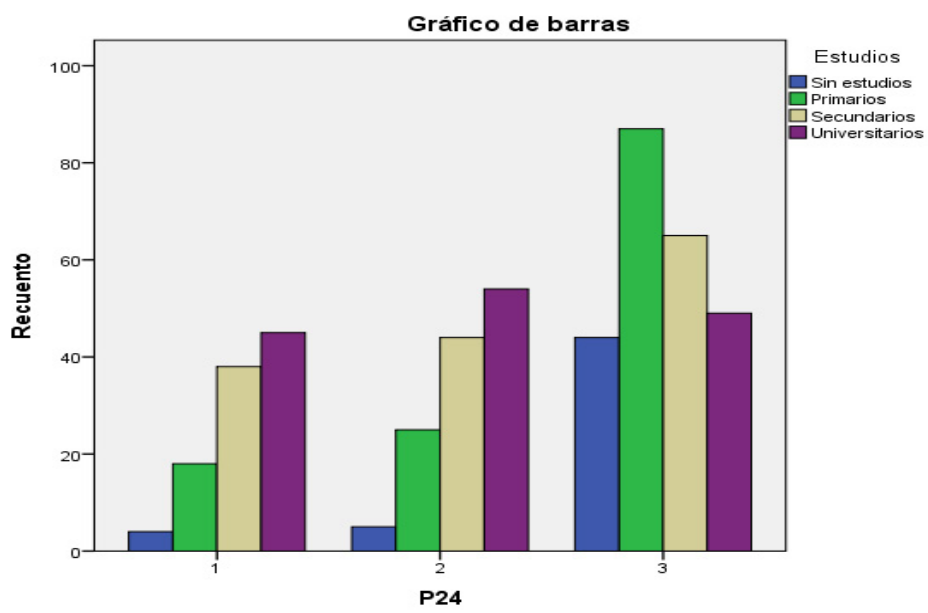

FUENTE: ELABORACIÓN PROPIA

Cuando preguntamos a los mayores de 55 años ¿Utiliza algún medio (e-mail, blog, etc.) para mandar mensajes, o realizar acciones que contribuyan a la mejora del entorno social en el que vive? Las respuestas, Sí, a menudo (1) y Sí, alguna vez (2), aumentan a medida que es mayor el nivel de estudios de los encuestados. El coeficiente de contingencia 32.6\% obtenido en el contraste Chi-cuadrado nos indica que es significativa la relación entre la difusión de valores y el nivel de estudios.

\subsubsection{INDICADORES DE LA DIMENSIÓN ESTÉTICA DE LA COMPETENCIA MEDIÁTICA}

La dimensión estética referida a las competencias mediáticas, con indicadores como sensibilidad para reconocer una producción mediática que no se adecue a unas exigencias mínimas de calidad estética, parece ser poco considerada en el caso de la población estudiada. En las respuestas al ítem 25 «A la hora de decidirse por un medio ¿tiene en cuenta sólo lo que comunica o en esa elección le da valor a los aspectos formales y estéticos?» no hemos encontrado resultados con un coeficiente de contingencia que sea significativo. 


\section{RESULTADOS Y CONCLUSIONES DEL ESTUDIO}

Los datos evidencian que las TICs al servicio del mayor, pueden ofrecer aspectos muy enriquecedores, tanto a nivel personal como para su integración social. Destacamos en primer lugar que, las personas mayores al avanzar en competencias mediáticas, pueden mejorar su comunicación, la más importante de las capacidades de los seres humanos. Una de las consecuencias más implícitas que lleva consigo la idea de jubilación es la de soledad física o psíquica ya que a esa edad también suele producirse la emancipación de los hijos. Pensamos que el desarrollo de las competencias mediáticas en los mayores puede:

- Facilitar la interacción entre los mayores. Está comprobado que la lucha contra el aislamiento y la soledad es un seguro de ralentización de la vejez. De esta manera, se puede conseguir la mejor realización personal. La interactividad es la característica fundamental de Internet y de la informática.

- Mejorar la autoestima. Estamos acostumbrados a oír de las personas de edad avanzada la típica frase: "Yo soy demasiado mayor para...», y es necesario concienciarlos de que llegar a viejos es lo mejor que nos puede pasar en esta vida.

- Propiciar una mayor participación social. «Vivir volcados al exterior» salvo en los trenes, deberíamos estar permanentemente mirando hacia fuera. El sistema capitalista, gobernado por la tiranía de lo urgente, no deja mucho sitio en el juego social para aquellos que han entrado ya en la jubilación.

- Mejorar la relación intergeneracional. Nuestros mayores son: «Memoria del pasado para iluminar el futuro»; no podemos olvidarnos de muchas cosas, pero debemos mirar al pasado de la misma forma que lo hacemos en el retrovisor del coche: «el tiempo suficiente para conducir bien mirando hacia delante».

En segundo lugar, y en cuanto a los resultados del estudio realizado, podemos concluir: Respecto al desarrollo de las competencias mediáticas en los mayores, y según el estudio realizado en la Comunidad Autónoma de Andalucía, habría que seguir incidiendo en mejorar la habilidad de los mayores para interactuar de forma significativa con los medios, ya que el aprovechamiento de las posibilidades que ofrece Internet, disminuye significativamente con la edad. Los mayores de 55 años sienten desconfianza ante las operaciones relacionadas con compras o servicios electrónicos, y en con- 
trapartida se observa gran interés y nivel de utilización de este colectivo en aplicaciones informáticas como correo electrónico, OpenOffice y Facebook.

En cuanto a la actitud activa en la interacción con las pantallas como medio para el desarrollo integral y como oportunidad para transformar y mejorar el entorno, se observa que hay una relación significativa y directa entre el nivel de estudios de los mayores y el compromiso adquirido en este aspecto con la comunidad. De la misma manera, la capacidad de aprovechar las TIC para transmitir valores y mostrar una actitud de compromiso social y cultural, también está en relación directa con el nivel de estudios de los mayores.

Por último, sería necesario aprovechar las tecnologías de la información como medio para favorecer la integración y comunicación en la población mayor, comprobados sus efectos positivos, de carácter integral, ya que el desarrollo de habilidades para interacturar de manera positiva con los medios permite a los mayores desarrollar sus capacidades mentales.

En el ámbito de la expresión deberíamos tener en cuenta que la capacidad de manejar correctamente herramientas comunicativas en un entorno multimedial conduce a la integración plena del mayor en nuestra sociedad.

Los Programas de Mayores dependientes de las Universidades (Bru y López, 2014), deberían implementar de forma consensuada el desarrollo de las competencias mediáticas entre el alumnado mayor, contribuyendo así a paliar las diferencias socioeconómicas existentes en el acceso a las TIC y a la comunicación multimedial, que han quedado de manifiesto en el estudio realizado.

\section{REFERENCIAS}

AGUADED, IGNACIO (2009). "El Parlamento Europeo apuesta por la alfabetización mediática”. Revista Comunicar, número 16. Huelva: Grupo Comunicar, pp. 7-8.

AGUADED, IGNÁCIO (2011). El grado de competencia mediática en la ciudadanía andaluza. [O grau de competência para os meios de comunicação entre os cidadãos andaluzes]. Huelva: Grupo Comunicar Ediciones/Ágora, Universidad de Huelva http://issuu.com/grupo-comunicar/docs/competencia-mediatica (Última consulta: 17 de marzo de 2015)

AGUADED, IGNACIO Y CRUZ-DIAZ, ROCÍO (2014). "O grau de competência em comunicação audiovisual entre os cidadãos da Andaluzia (Espanha)". Revista Comunicação \& Educação, número 19. Brasil: Universidad de São Paulo, pp. 67-72. http://goo.gl/vdk3fy (http://dx.doi.org/10.11606/ 
issn.2316-9125.v19i1p67-72) (Última consulta: 9 de abril de 2015).

AGULLO TOMAS, SILVERIA (2001). Mayores, actividad y trabajo en el proceso de envejecimiento y jubilación: una aproximación psico-sociológica. Madrid. IMSERSO.

BRU RONDA, CONCEPCIÓN. Y LÓPEZ, ANGEL (2014).Uso de las TIC en los programas Universitarios para personas mayores. Universidad de Alicante. Disponible: http://goo.gl/SxSsHL (Última consulta: 27 de abril de 2015).

CASTELLS, MANUEL. (2001). La galaxia Internet. Madrid: Areté.

COMISIÓN EUROPEA (2000). COM(2000 318final). Programa e-learning. Concebir la educación del futuro. Lisboa: UE. http://www.usc.es/tecnoeduc/ docs/comision_europea_2000.pdf (Última consulta: 27 de abril de 2015)

COMISIÓN EUROPEA (2013). Programa de Aprendizaje Permanente (PAP/ LLLP 2007/2013). Bruselas. UE. http://www.oapee.es/dctm/weboapee/ servicios/documentos/2013/part1es.pdf?documentId=0901e72b8145e8e6 (Última consulta: 27 de abril de 2015).

CONTRERAS PULIDO, PALOMA, MARFIL CARMONA, RAFAEL Y ORTEGA, JUANA MARÍA (2014). "La competencia mediática de las perso $\urcorner$ nas mayores andaluzas: retos para una inclusión social plena". Revista Historia y Comunicación Social. Vol. 19. Madrid: Universidad Complutense de Madrid. pp. 129-142. (http://dx.doi.org/10.5209/rev_HICS.2014.v19.44946) CRUZ DÍAZ, ROCÍO, MORENO-CRESPO, PILAR Y REBOLLEDO GÁMEZ, TERESA (2013). "Formación universitaria de mayores. Un análisis del Aula Abierta de Mayores desde la perspectiva del alumnado". Revista Hekademos, 14, Año VI, Diciembre. Sevilla: AFOE. pp. 41-51.

CRUZ DÍAZ, ROCÍO Y ACOSTA SORIANO, SARAY (2010). "Vocabulario intergeneracional. Un intercambio entre mayores y alumnos en las aulas". Revista Cuestiones Pedagógicas, número 20. Sevilla: Universidad de Sevilla. pp. 247-268.

CRUZ DÍAZ, ROCÍO (2003). "La comunicación no verbal en contextos educativos. De lo que dicen las miradas". Revista Comunicar, número 20. Huelva: Grupo Comunicar. pp. 188-194.

FERRÉS, JOAN (2006). "La competencia en comunicación audiovisual: propuesta articulada de dimensiones e indicadores". Revista Quaderns del CAC, número 25. Barcelona: Consell de l'Audiovisual de Catalunya, pp. 9-18.

FERRÉS, JOAN (2007). "La competencia en comunicación audiovisual: dimensiones e indicadores". Revista Comunicar, número 29. Huelva, Grupo Comunicar, pp. 100-107.

FERRÉS, JOAN et al. (2011). Competencia mediática, Investigación sobre el grado de competencia de la ciudadanía en España. Madrid. Instituto de Tecnología 
Educativa. Ministerio de educación.

FERRÉS, JOAN Y PISCITELLI, ALEJANDRO (2012). "La competencia mediática: propuesta articulada de dimensiones e indicadores”. Revista Comunicar, número 38. Huelva, Grupo Comunicar, pp. 75-82. (DOI: 10.3916/C382012-02-08).

GARCÍA ARROYO, MARIA JESÚS (2001). "La participación educativa de las personas mayores y la calidad de vida". En Actas del III Encuentro Nacional de Programas Universitarios para Personas Mayores. Salamanca. Publicaciones Universidad Pontificia de Salamanca.

GUTIÉRREZ MARÍN, ALFONSO. Y TYNER, KATHLEEN (2012). "Educación para los medios, alfabetización mediática y competencia digital". Revista Comunicar, número 38. Huelva: Grupo Comunicar, pp. 31-39. (DOI: 10.3916/C38-2012-02-03).

MACÍAS GONZÁLEZ, LIBERTO Y MANRESA YEE, CRISTINA. (2013). "Mayores y nuevas tecnologías: motivaciones y dificultades". Revista Ariadna; cultura, educación y tecnología número 1, Castellón: Universidad Jaume I, pp. 6-11. DOI: http://dx.doi.org/10.6035/Ariadna.2013.1.13

MARTÍNEZ, ROBERTO, CABECINHAS, ROSA Y LOSCERTALES, FELICIDAD (2011). "Mayores Universitarios en la Red". Revista Comunicar, número 37. Huelva: Grupo Comunicar, pp. 89-95. (DOI: 10.3916/C37-201102-09).

PAVÓN, FRANCISCO (2000). "Tecnologías avanzadas: nuevos retos de comunicación para los mayores". Revista Comunicar, número 15, Huelva: Grupo Comunicar, pp. 133-139.

PAVÓN, FRANCISCO Y CASANOVA, JUAN (2008). "Enseñar desde la escuela y la familia a ver TV”. Revista Comunicar, número 31, Huelva: Grupo Comunicar, pp. 265-268. (DOI: 10.3916/c31-2008-03-004)

PAVÓN, FRANCISCO Y CASANOVA, JUAN (2006). "Telefonía móvil y personas mayores: la accesibilidad como derecho". Revista RELATEC Revista latinoamericana de tecnología educativa, Número 5, Vol 2, pp. 385-395.

RAMÍREZ GARCÍA, ANTONIA, RENÉS ARELLANO, PAULA Y SÁNCHEZ CARRERO, JAQUELINE (2013). "Educación artística y competencia mediática en el curriculum de Educación primaria”. Revista Historia y Comunicación Social, Vol. 8. Número Especial octubre, Madrid. Universidad Complutense, pp. 673-686.

VILAPLANA PRIETO, CRISTINA (2013). “¿Es la jubilación un buen momento para aprender informática?". Revisa Ariadna; cultura, educación y tecnología, número 1, Castellón: Universidad Jaume I. pp. 77-82. (DOI: http://dx. doi.org/10.6035/Ariadna.2013.1.13) 